\title{
Vitamins and lung cancer
}

\author{
Jarmo Virtamo \\ National Public Health Institute, Mannerheimintie 166, FIN-00300 Helsinki, Finland
}

\begin{abstract}
Lung cancer is the most common cancer in the world, and smoking is the major risk factor, accounting for abo ut $90 \%$ of the cases. Diet has also been implicated in the development of lung cancer, although the specific nutrients remain to be elucidated. Vitamins with antioxidant activity have received much attention. $\beta$-Carotene, the most efficient provitamin $\mathrm{A}$, was found to be inversely related to the risk of lung cancer in many prospective epidemiological studies, especially in studies measuring serum concentrations of $\beta$-carotene. The findings from controlled trials, however, contradict the hypothesis that $\beta$-carotene could prevent lung cancer, but rather suggest increased risk of lung cancer with supplementary $\beta$-carotene. Data from both prospective studies and a controlled trial suggest no role for vitamin $\mathrm{E}$ in lung carcinogenesis. Some prospective epidemiological studies suggest an inverse relationship between dietary vitamin $\mathrm{C}$ and the risk of lung cancer, but due to the high correlation between dietary vitamin $\mathrm{C}$ and vegetable and fruit intake the independent role of dietary vitamin $\mathrm{C}$ is difficult to estimate. Studies using prediagnostic plasma concentrations of ascorbic acid do not support the involvement of vitamin $\mathrm{C}$ in lung carcinogenesis, and no controlled trials of vitamin $\mathrm{C}$ on lung cancer have been published. Thus, supplementation with antioxidant vitamins cannot be recommended for the prevention of lung cancer. Non-smoking is the most important target in the prevention of lung cancer. High i ntakes of vegetables and fruits may provide additional protection and are unlikely to be harmful.
\end{abstract}

Lung cancer: Antioxidants: $\beta$-Carotene: Vitamin E: Vitamin C

Lung cancer is the most common cancer in the world. In 1996, an estimated 1.3 million new cases were diagnosed worldwide, accounting for $12.8 \%$ of all new cases of cancer (World Cancer Research Fund and American Institute for Cancer Research, 1997). Prevention, early detection and treatment are the tools to reduce lung cancer morbidity and mortality. Early detection of lung cancer has not been successful, as symptoms often do not appear until the disease is advanced. Similarly, screening of asymptomatic subjects with regular chest x-rays or sputum cytology has not been successful. Despite advances in the treatment of lung cancer, the 5-year survival for lung cancer is only $10-15 \%$. Thus, the only efficient way to reduce the burden from lung cancer is prevention.

Cigarette smoking is the predominant risk factor for lung c ancer; about $90 \%$ of the cases are attributable to cigarette smoking. Encouraging non-smokers not to start and current smokers to quit is of primary importance in reducing lung cancer incidence and mortality. Other risk factors for lung cancer include passive smoking, asbestos, radon, chemical carcinogens, previous chronic inflammatory lung disease and genetic predisposition.

Diet has also been implicated in the development of lung cancer. Despite the extensive research conducted in this area, the relationship between diet and lung cancer is still not clear. Diets high in fat and low in vegetables and fruits may increase the risk of lung cancer (Ziegler et al. 1996). The specific mechanisms of the interaction between diet and lung cancer remain to be elucidated.

\section{Vitamin $A$ and $\beta$-carotene}

Experiments with animal models have demonstrated that high doses of retinoids could inhibit carcinogenesis in the respiratory tract (Moon et al. 1994), whereas $\beta$-carotene has been ineffective (IARC Working Group on the Evaluation of Cancer Preventive Agents, 1998). Early studies of diet and lung cancer in human populations evaluated the importance of vitamin A. In a cohort of Norwegian men, total vitamin A intake was significantly 
associated with reduced lung cancer risk after adjustment for smoking (Bjelke, 1975; Kvåle et al. 1983). In this study, milk and carrots contributed substantially to vitamin A intake, and each food item was related strongly and inversely to the risk of lung cancer. An early cohort study in Japan (Hirayama, 1979) found that daily consumption of green or yellow vegetables rich in carotenoids was associated significantly with reduced lung cancer risk compared with more infrequent consumption. In the first cohort study evaluating the independent contributions of dietary carotene and retinol, reduced lung cancer risk was associated only with increased intake of carotene (Shekelle et al. 1981). Thereafter, eight prospective studies have reported on $\beta$ carotene intake and most of them have suggested a nonsignificant association in the direction of decreased risk of lung cancer (Kromhout, 1987; Knekt et al. 1991; Chow et al. 1992; Shibata et al. 1992b; Steinmetz et al. 1993; Bandera et al. 1997; Ocké et al. 1997; Yong et al. 1997).

Several cohort studies have stored serum or plasma, and later determined $\beta$-carotene concentration. Eight studies have demonstrated that prediagnostic serum or plasma $\beta$-carotene levels, (measured by HPLC) were significantly lowered in participants who developed or died from lung cancer (Nomura et al. 1985; Menkes et al. 1986; Wald et al. 1988; Connett et al. 1989; Knekt et al. 1990; Stähelin et al. 1991; Orentreich et al. 1991; Comstock et al. 1997). Examination of the relationship over time suggested that preclinical disease was not responsible for the effect. The prediagnostic blood retinol level has not been associated with the incidence of lung cancer in prospective studies.

Numerous prospective and retrospective studies have evaluated the role of vegetables and fruits in the aetiology of lung cancer (Steinmetz \& Potter, 1991a). In the great majority of these studies, lung cancer risk was reduced at high levels of vegetable and/or fruit consumption. Associations found in cohort studies are, however, less strong than those in case-control studies (Koo, 1997). Some studies have evaluated both $\beta$-carotene intake and vegetable and fruit intake, and in many of these notably stronger inverse trends with vegetable and fruit intake have been found than with estimated $\beta$-carotene intake. Thus, $\beta$-carotene does not explain completely the association of vegetable and fruit consumption with reduced lung cancer risk observed in epidemiological studies. Thus, other compounds in vegetables and fruits seem to be involved (e.g. flavonoids, phenols, indoles, dithiolthiones and isothiocyanates), which, on the basis of animal and in vitro experiments, might be important in human cancer prevention (Steinmetz \& Potter, 1991b).

The association of the main dietary carotenoids with lung cancer risk has been examined in two case-control studies (Le Marchand et al. 1993; Ziegler et al. 1996); dietary $\alpha$-carotene and lutein but not lycopene and $\beta$-cryptoxanthin were inversely associated with lung cancer incidence, but in both studies a high intake of vegetables was associated with reduced lung cancer risk more strongly than a high intake of the individual carotenoids. No significant associations between dietary $\alpha$-carotene, $\beta$-carotene, lutein and lycopene and the risk of lung cancer were observed in two prospective studies (Knekt et al. 1991; Steinmetz et al. 1993). In the only prospective serum study, serum concen- trations of cryptoxanthin, $\beta$-carotene and lutein were significantly lower among lung cancer cases than controls, a modest non-significant difference in a protective direction was noted for $\alpha$-carotene and no difference for lycopene (Comstock et al. 1997).

Peto et al. (1981), noting the protective effects of increased vegetable and fruit consumption in epidemiological studies of lung and other cancers, speculated that $\beta$-carotene, the most abundant and efficiently converted of the provitamin A carotenoids in vegetables and fruits, might protect against cancer. On the basis of the consistency of the epidemiological findings and the evidence for tumour inhibition in animal models, controlled clinical trials were initiated in the 1980s to evaluate the potential of $\beta$-carotene in the prevention of lung and other cancers. The mechanism of chemoprevention of lung cancer by supplementary $\beta$-carotene was not known, but some of the known $\beta$-carotene functions were thought to be involved, e.g. its role as an antioxidant, in enhancing immune function, and as a precursor to vitamin A.

Primary prevention trials of lung cancer enrol ideally 20000-30000 high-risk subjects, heavy smokers or asbestos-exposed subjects. These trials require many years of intervention, and are expensive and laborious to complete. In recent years, three major intervention trials have been completed, all of which included supplementation with $\beta$-carotene, with or without other nutrients. The AlphaTocopherol, Beta-Carotene Cancer Prevention Study (ATBC) was a randomized double-blind placebo-controlled primary-prevention trial to test whether daily supplementation with $\alpha$-tocopherol, $\beta$-carotene, or both, would reduce the incidence of lung cancer (The ATBC Cancer Prevention Study Group, 1994; The Alpha-Tocopherol, Beta Carotene Cancer Prevention Study Group, 1994). A total of 29133 male smokers aged 50-69 years from southwestern Finland were randomly assigned to receive either $\alpha$-tocopherol $(50 \mathrm{mg} / \mathrm{d})$ alone, $\beta$-carotene $(20 \mathrm{mg} / \mathrm{d})$ alone, both $\alpha$-tocopherol and $\beta$-carotene, or a placebo, for $5-8$ (median 6.1) years. The Beta-Carotene and Retinol Efficacy Trial (CARET) enrolled 18314 men and women at high risk for lung cancer because of a history of heavy cigarette smoking or occupational exposure to asbestos (Omenn et al. 1996b). This trial evaluated combined supplementation with $\beta$-carotene $(30 \mathrm{mg} / \mathrm{d})$ and retinol $(7.5 \mathrm{mg} / \mathrm{d}) v$. placebo for an average of 4 years. The Physicians' Health Study (PHS) was a randomized doubleblind placebo-controlled trial of $\beta$-carotene $(50 \mathrm{mg}$ on alternate days) supplementation for 12 years among 22071 male physicians who were 40-84 years of age (Hennekens et al. 1996).

In ATBC 894 new cases of lung cancer were diagnosed during follow-up. The relative risk of lung cancer was $1.16(95 \%$ CI 1.02, 1.33) among subjects who received $\beta$-carotene supplementation compared with those who received no supplement (Albanes et al. 1996). In CARET a total of 388 new cases of lung cancer were identified, and the relative risk of lung cancer was 1.28 (95\% CI 1.04, 1.57 ) among those receiving the daily combination of $\beta$-carotene and retinol compared with those receiving the placebo (Omenn et al. 1996a). In the PHS there was no significant difference in the number of cases of lung 
cancer (eighty-two cases in the $\beta$-carotene group $v$. eighty-eight cases in the placebo group; Hennekens et al. 1996).

Thus, the groups receiving $\beta$-carotene supplementation had an increased risk for lung cancer in both ATBC and CARET. No change in lung cancer risk was found in PHS, but its potential to detect a small change in lung cancer risk was limited by the relatively small number of cases of lung cancer. It is worth noting that $\beta$-carotene supplementation did not influence significantly the incidence of any other cancer in these studies. Differences in the study designs may explain some of the divergent results. The dose of supplementary $\beta$-carotene varied from 15 to $30 \mathrm{mg} / \mathrm{d}$ in the trials. The study populations also varied in their serum responses to supplementary $\beta$-carotene. The highest serum concentrations of $\beta$-carotene during supplementation were observed in ATBC and CARET (medium 3.0 and $2.1 \mathrm{mg} / \mathrm{l}$ respectively), whereas notably lower concentrations were found in the PHS (mean $1.2 \mathrm{mg} / \mathrm{l}$ ).

In ATBC all the participants were smokers, in CARET $60 \%$ were smokers, and in PHS only $11 \%$ were smokers. The findings of CARET suggested increased risks for lung cancer among current smokers and asbestos-exposed workers, but not among former smokers. In ATBC, the increased risk for lung cancer appeared to be confined to people smoking at least twenty cigarettes daily, and no effect on risk was found for those smoking five to nineteen cigarettes daily (although the test for effect modification was not statistically significant). Long-term cigarette smoking did not significantly modify the effect of $\beta$-carotene supplementation on lung cancer risk. These findings indicate that $\beta$-carotene supplementation accelerates the clinical appearance of lung cancer among current heavy smokers. The findings of ATBC and CARET suggested that alcohol intake may modify the effect of $\beta$-carotene on lung cancer. The alcohol intake levels were, however, moderate; the median in ATBC was $11 \mathrm{~g} / \mathrm{d}$ and that in CARET $3 \mathrm{~g} / \mathrm{d}$. In $\mathrm{ATBC}$, the increase in lung cancer risk with supplementary $\beta$-carotene appeared mainly in those reporting alcohol consumption above the median. CARET findings also suggested that the risk for lung cancer was greater in participants with a high alcohol intake (>18.7 g/d), but there was no consistent dose-response relationship. The separate effects of alcohol drinking and tobacco smoking are, however, difficult to distinguish, as the two behaviours are correlated.

Mechanisms to explain the higher incidence of lung cancers observed in participants receiving $\beta$-carotene supplements in ATBC and CARET are speculative. The combination of tobacco smoke, which contains many free radicals and is strongly oxidative, together with relatively high partial pressures of $\mathrm{O}_{2}$ in the lung may trigger autooxidation of $\beta$-carotene in the lung. Under such conditions, the free radical of $\beta$-carotene may also serve as a propagator of free-radical formation. Transformed or damaged cells found in the lungs of long-term smokers might be particularly sensitive to either modulation of the oxidative state or the non-physiological concentrations of $\beta$-carotene present (IARC Working Group on the Evaluation of Cancer Preventive Agents, 1998).

\section{Vitamin E}

The association between dietary intake of vitamin $\mathrm{E}$ and lung cancer has been evaluated only in a few prospective studies, and the results have varied from a weak inverse association to no association (Connett et al. 1989; Knekt et al. 1991; Bandera et al. 1997; Ocké et al. 1997; Yong et al. 1997). Cohort studies of serum concentrations of $\alpha$-tocopherol show no significant associations with lung cancer (Willett et al. 1984; Nomura et al. 1985; Kok et al. 1987; Wald et al. 1987; Knekt et al. 1988; Connett et al. 1989; Orentreich et al. 1991; Stähelin et al. 1991; Comstock et al. 1997), except one that found a significant inverse association (Menkes et al. 1986).

Only one controlled trial has reported results on the effect of supplementary vitamin $\mathrm{E}$ ( $\alpha$-tocopherol) on the risk of lung cancer. In ATBC, 14564 men received $\alpha$-tocopherol supplementation of $50 \mathrm{mg} / \mathrm{d}$ and $14569 \mathrm{did}$ not receive $\alpha$-tocopherol supplementation, for 5-8 (median 6.1) years, with no overall effect for lung cancer from $\alpha$-tocopherol supplementation (relative risk 0.99 (95\% CI 0.87, 1.13); Albanes et al. 1996).

\section{Vitamin C}

Six prospective studies have developed indices of vitamin C intake from vegetables and fruits and studied the association between these indices and lung cancer risk. The results have varied from an inverse association to no association (Kvåle et al. 1983; Kromhout, 1987; Knekt et al. 1991; Chow et al. 1992; Shibata et al. 1992a; Steinmetz et al. 1993), but three of the most recent reports have demonstrated a significant inverse association between dietary vitamin $\mathrm{C}$ and the risk of lung cancer (Bandera et al. 1997; Ocké et al. 1997; Yong et al. 1997). An association between plasma ascorbic acid concentration and lung cancer risk has been reported in two studies; in one study plasma ascorbic acid was not predictive of subsequent lung cancer mortality (Stähelin et al. 1991), and in the other study a modest non-significant protection was noted (Comstock et al. 1997). No controlled trials of supplementation with vitamin $\mathrm{C}$ in relation to lung cancer have been published.

\section{Summary and conclusions}

Observational studies of diet and lung cancer suggest strongly that increased vegetable and fruit intake is associated with reduced risk of lung cancer. Prospective studies of blood $\beta$-carotene levels indicate that low levels are predictive of increased lung cancer incidence. The findings from the controlled clinical trials, however, do not support the hypothesis that supplementation with $\beta$-carotene or $\alpha$-tocopherol would reduce the incidence of lung cancer. In contrast, there are suggestions that supplementation with $\beta$-carotene may increase the incidence of lung cancer among smokers, the group with the highest risk of lung cancer. Thus, supplementary vitamins cannot be recommended for prevention of lung cancer.

Avoidance of cigarette smoking is the most effective method of reducing the risk of lung cancer. Quitting 
smoking causes approximately a 10-fold drop in the risk, whereas the decrease in the risk of lung cancer associated with increased vegetable and fruit intake is, at most, 2-fold. However, a diet rich in vegetables and fruits containing many potentially-beneficial nutrients and phytochemicals is to be recommended in order to reduce the risk of lung cancer and also other chronic diseases, and certainly such dietary modification is unlikely to be harmful.

\section{References}

Albanes D, Heinonen OP, Taylor PR, Virtamo J, Edwards BK, Rautalahti M, Hartman AM, Palmgren J, Freedman LS, Haapakoski J, Barrett MJ, Pietinen P, Malila N, Tala E, Liippo K, Salomaa ER, Tangrea JA, Teppo L, Askin FB, Taskinen E, Erozan Y, Greenwald P \& Huttunen J (1996) $\alpha$-Tocopherol and $\beta$-carotene supplements and lung cancer incidence in the Alpha-Tocopherol, Beta-Carotene Cancer Prevention Study: effects of base-line characteristics and study compliance. Journal of the National Cancer Institute 88, $1560-1570$.

Bandera EV, Freudenheim JL, Marshall JR, Zielezny M, Priore RL, Brasure J, Baptiste M \& Graham S (1997) Diet and alcohol consumption and lung cancer risk in the New York State Cohort (United States). Cancer Causes and Control 8, 828-840.

Bjelke E (1975) Dietary vitamin A and human lung cancer. International Journal of Cancer 15, 561-565.

Chow WH, Schuman LM, McLaughlin JK, Bjelke E, Gridley G, Wacholder S, Chien HTC \& Blot WJ (1992) A cohort study of tobacco use, diet, occupation, and lung cancer mortality. Cancer Causes and Control 3, 247-254.

Comstock GW, Alberg AJ, Huang HY, Wu K, Burke AE, Hoffman SC, Norkus EP, Gross M, Cutler RG, Morris JS, Spate VL \& Helzlsouer KJ (1997) The risk of developing lung cancer associated with antioxidants in the blood: ascorbic acid, carotenoids, $\alpha$-tocopherol, selenium, and total peroxyl radical absorbing capacity. Cancer Epidemiology, Biomarkers and Prevention 6, 907-916.

Connett JE, Kuller LH, Kjelsberg MO, Polk BF, Collins G, Rider A \& Hulley SB (1989) Relationship between carotenoids and cancer: the Multiple Risk Factor Intervention Trial (MRFIT) Study. Cancer 64, 126-134.

Hennekens CH, Buring JE, Manson JE, Stampfer M, Rosner B, Cook NR, Belanger C, LaMotte F, Gaziano JM, Ridker PM, Willett W \& Peto R (1996) Lack of effect of long-term supplementation with beta carotene on the incidence of malignant neoplasms and cardiovascular disease. New England Journal of Medicine 334, 1145-1149.

Hirayama T (1979) Diet and cancer. Nutrition and Cancer 1, 6781.

IARC Working Group on the Evaluation of Cancer Preventive Agents (1998) IARC Handbooks of Cancer Prevention, vol. 2, Carotenoids. Lyon, France: International Agency for Research on Cancer.

Knekt P, Aromaa A, Maatela J, Aaran RK, Nikkari T, Hakama M, Hakulinen T, Peto R, Saxen E \& Teppo L (1988) Serum vitamin $\mathrm{E}$ and risk of cancer among Finnish men during a 10-year followup. American Journal of Epidemiology 127, 28-41.

Knekt P, Aromaa A, Maatela J, Aaran RK, Nikkari T, Hakama M, Hakulinen T, Peto R \& Teppo L (1990) Serum vitamin A and subsequent risk of cancer: cancer incidence follow-up of the Finnish mobile clinic health examination survey. American Journal of Epidemiology 132, 857-870.

Knekt P, Järvinen R, Seppänen R, Rissanen A, Aromaa A, Heinonen OP, Albanes D, Heinonen M, Pukkala E \& Teppo L (1991)
Dietary antioxidants and risk of lung cancer. American Journal of Epidemiology 134, 471-479.

Kok FJ, van Duijn CM, Hofman A, Vermeeren R, de Bruijn AM \& Valkenburg HA (1987) Micronutrients and the risk of lung cancer. New England Journal of Medicine 316, 1416.

Koo LC (1997) Diet and lung cancer 20+ years later: more questions than answers? International Journal of Cancer 10, Suppl., 22-29.

Kvåle G, Bjelke E \& Gart JJ (1983) Dietary habits and lung cancer risk. International Journal of Cancer 31, 397-405.

Kromhout D (1987) Essential micronutrients in relation to carcinogenesis. American Journal of Clinical Nutrition 45, 1361-1367.

Le Marchand L, Hankin JH, Kolonel LN, Beecher GR, Wilkens LR \& Zhao LP (1993) Intake of specific carotenoids and lung cancer risk. Cancer Epidemiology, Biomarkers and Prevention 2, 183-187.

Menkes MS, Comstock GW, Vuilleumier JP, Helsing KJ, Rider AA \& Brookmeyer R (1986) Serum beta-carotene, vitamins A and E, selenium, and the risk of lung cancer. New England Journal of Medicine 315, 1250-1254.

Moon RC, Mehta RG \& Rao KVN (1994) Retinoids and cancer in experimental animals. In The Retinoids: Biology, Chemistry, and Medicine, 2nd ed., pp. 573-595 [MB Sporn, AB Roberts and DS Goodman, editors]. New York: Raven Press.

Nomura AMY, Stemmermann GN, Heilbrun LK, Salkeld RM \& Vuilleumier JP (1985) Serum vitamin levels and the risk of cancer of specific sites in men of Japanese ancestry in Hawaii. Cancer Research 45, 2369-2372.

Ocké MC, Bueno-de-Mesquita HB, Feskens EJM, van Staveren WA \& Kromhout D (1997) Repeated measurements of vegetables, fruits, $\beta$-carotene, and vitamins $C$ and $E$ in relation to lung cancer. American Journal of Epidemiology 145, 358-365.

Omenn GS, Goodman GE, Thornquist MD, Balmes J, Cullen MR, Glass A, Keogh JP, Meyskens FL Jr, Valanis B, Williams JH Jr, Barnhart S, Cherniack MG, Brodkin CA \& Hammar S (1996a) Risk factors for lung cancer and for intervention effects in CARET, the Beta-Carotene and Retinol Efficacy Trial. Journal of the National Cancer Institute 88, 1550-1559.

Omenn GS, Goodman GE, Thornquist MD, Balmes J, Cullen MR, Glass A, Keogh JP, Meyskens FL Jr, Valanis B, Williams JH Jr, Barnhart S \& Hammar S (1996b) Effects of a combination of beta carotene and vitamin A on lung cancer and cardiovascular disease. New England Journal of Medicine 334, 1150-1155.

Orentreich N, Matias JR, Vogelman JH \& Salkeld RM (1991) The predictive value of serum $\beta$-carotene for subsequent development of lung cancer. Nutrition and Cancer 16, 167-168.

Peto R, Doll R, Buckley JD \& Sporn MB (1981) Can dietary betacarotene materially reduce human cancer rates? Nature 290, 201-208.

Shekelle RB, Lepper M, Liu S, Maliza C, Raynor WJ Jr, Rossof AH, Paul O, Shryock AM \& Stamler J (1981) Dietary vitamin A and risk of cancer in the Western Electric Study. Lancet ii, $1185-1190$.

Shibata A, Paganini-Hill A, Ross RK \& Henderson BE (1992a) Intake of vegetables, fruits, beta-carotene, vitamin $\mathrm{C}$ and vitamin supplements and cancer incidence among the elderly: a prospective study. British Journal of Cancer 66, 673-679.

Shibata A, Paganini-Hill A, Ross RK, Yu MC \& Henderson BE (1992b) Dietary beta carotene, cigarette smoking, and lung cancer in men. Cancer Causes and Control 3, 207-214.

Stähelin HB, Gey KF, Eichholzer M, Lüdin E, Bernasconi F, Thurneysen J \& Brubacher G (1991) Plasma antioxidant vitamins and subsequent cancer mortality in the 12-year followup of the prospective Basel Study. American Journal of Epidemiology 133, 766-775. 
Steinmetz KA \& Potter JD (1991a) Vegetables, fruit, and cancer. I. Epidemiology. Cancer Causes and Control 2, 325-357.

Steinmetz KA \& Potter JD (1991b) Vegetables, fruit, and cancer. II. Mechanisms. Cancer Causes and Control 2, 427-442.

Steinmetz KA, Potter JD \& Folsom AR (1993) Vegetables, fruit, and lung cancer in the Iowa Women's Health Study. Cancer Research 53, 536-543.

The Alpha-Tocopherol, Beta Carotene Cancer Prevention Study Group (1994) The effect of vitamin E and beta carotene on the incidence of lung cancer and other cancers in male smokers. New England Journal of Medicine 330, 1029-1035.

The ATBC Cancer Prevention Study Group (1994) The AlphaTocopherol, Beta-Carotene Lung Cancer Prevention Study: design, methods, participant characteristics, and compliance. Annals of Epidemiology 4, 1-10.

Wald NJ, Thompson SG, Densem JW, Boreham J \& Bailey A (1987) Serum vitamin E and subsequent risk of cancer. British Journal of Cancer 56, 69-72.

Wald NJ, Thompson SG, Densem JW, Boreham J \& Bailey A (1988) Serum beta-carotene and subsequent risk of cancer: results from the BUPA Study. British Journal of Cancer 57, 428-433.

Willett WC, Polk BF, Underwood BA, Stampfer MJ, Pressel S, Rosner B, Taylor JO, Schneider K \& Hames CG (1984) Relation of serum vitamins $\mathrm{A}$ and $\mathrm{E}$ and carotenoids to the risk of cancer. New England Journal of Medicine 310, 430-434.

World Cancer Research Fund and American Institute for Cancer Research (1997) Food, Nutrition and the Prevention of Cancer: A Global Perspective. Washington, DC: AICR.

Yong LC, Brown CC, Schatzkin A, Dresser CM, Slesinski MJ, Cox CS \& Taylor PR (1997) Intakes of vitamins E, C, and A and risk of lung cancer. The NHANES I Epidemiologic Followup Study. American Journal of Epidemiology 146, 231-243.

Ziegler RG, Colavito EA, Hartge P, McAdams MJ, Schoenberg JB, Mason TJ \& Fraumeni JF Jr (1996) Importance of $\alpha$-carotene, $\beta$-carotene, and other phytochemicals in the etiology of lung cancer. Journal of the National Cancer Institute 88, 612-615.

Ziegler RG, Mayne ST \& Swanson CA (1996) Nutrition and lung cancer. Cancer Causes and Control 7, 157-177. 
https://doi.org/10.1017/S0029665199000440 Published online by Cambridge University Press 